J. Phys. IV France 127 (2005) 119-124

(C) EDP Sciences, Les Ulis

DOI: $10.1051 / \mathrm{jp} 4: 2005127018$

\title{
Mesure de coefficients d'absorption de plasmas créés par laser nanoseconde
}

\author{
F. Thais ${ }^{1}$, C. Chenais-Popovics ${ }^{2}$, K. Eidmann ${ }^{3}$, S. Bastiani ${ }^{2}$, T. Blenski ${ }^{1}$ \\ et F. Gilleron ${ }^{4}$ \\ ${ }^{1}$ CEA/DSM, Saclay, 91191 Gif-sur-Yvette Cedex, France \\ ${ }^{2}$ LULI, UMR No. 7605 CNRS - École Polytechnique - CEA - Université Paris VI, \\ 91128 Palaiseau Cedex, France \\ ${ }^{3}$ Max-Planck-Institut für Quantenoptik, 85748 Garching, Allemagne \\ ${ }^{4}$ CEA/DAM, BP. 12, 91680 Bruyères-le-Châtel Cedex, France
}

\begin{abstract}
Résumé. La mesure des coefficients d'absorption dans les plasmas chauds est particulièrement utile dans le domaine de la fusion par confinement inertiel ainsi que dans divers contextes en astrophysique. Le développement des calculs de physique atomique qui y sont associés repose sur des hypothèses qu'il est nécessaire de vérifier dans la plus large gamme possible de conditions physiques. Nous présentons ici la méthode de mesure et d'analyse employée en nous appuyant sur l'exemple des cibles multicouches nickel/aluminium.
\end{abstract}

\section{INTRODUCTION}

La connaissance des coefficients de photoabsorption est essentielle pour le calcul du transfert radiatif dans les plasmas chauds et denses, aussi bien les plasmas considérés à l'équilibre thermodynamique, que hors de cet équilibre [1]. La fusion par confinement inertiel (FCI) et l'astrophysique sont deux domaines pour lesquels le transfert radiatif est particulièrement important. Par exemple, on sait que les modèles d'évolution solaire ont des résultats fortement corrélés aux coefficients d'absorption de corps à numéro atomique de Z moyen, bien que ceux-ci soient très minoritaires par rapport à l'hydrogène et à l'hélium [2] . Dans un autre domaine de conditions physiques une structure d'absorption du fer (figure 1), connue par expérience laser, a été récemment identifiée dans un quasar [3, 4]. Pour la FCI, le calcul du transfert d'énergie vers la cible fusible proprement dite est fortement dépendant de l'opacité des différents milieux rencontrés [5]. La photo-absorption est par ailleurs un moyen important de diagnostic des plasmas de laboratoire.

Lors de la conduite d'expériences avec des lasers de haute énergie, on utilise successivement la mesure de transmission dans différents corps. Dans un matériau à $\mathrm{Z}$ faible, comme l'aluminium, les mesures sont centrées sur des transitions bien connues, de type $1 s-2 p$, très sensibles à la température, et qui jouent donc un rôle de «thermomètre» du milieu. Le nickel est ensuite utilisé comme corps à $\mathrm{Z}$ moyen notamment parce qu'il a un numéro atomique proche de celui du fer et qu'il est disponible sous forme de couches minces de bonne homogénéité. Un «mélange» de ces deux composants, sous forme de multi-couches $\mathrm{Al} / \mathrm{Ni}$, permet de combiner les informations recueillies, c'est à dire d'aborder la mesure des corps à $\mathrm{Z}$ moyen tout en ayant un diagnostic de température. Nous avons par ailleurs prolongé cette étude en utilisant une technique de confinement de l'échantillon par des couches de carbone afin d'explorer le plasma à différentes densités [6].

e-mail : frederic.thais@cea.fr 


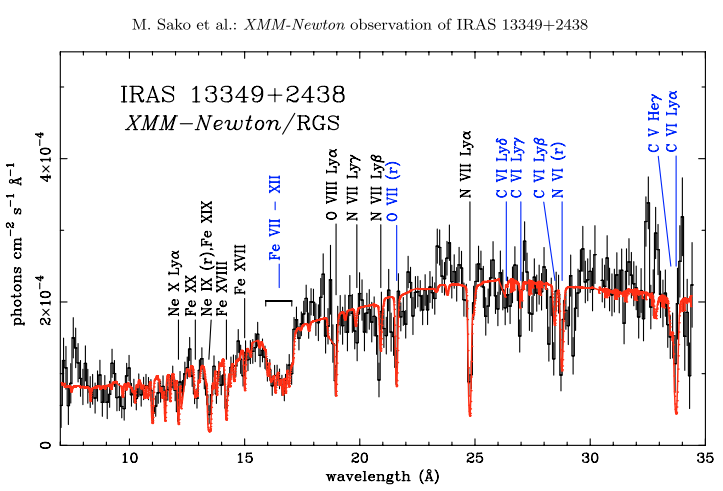

Figure 1. Structure d'absorption du fer VII-XII reconnue dans le spectre X du quasar IRAS 13349+2438.

\section{MÉTHODE EXPÉRIMENTALE}

Pour les expériences réalisées au LULI que nous prenons comme exemple, on utilise deux faisceaux du

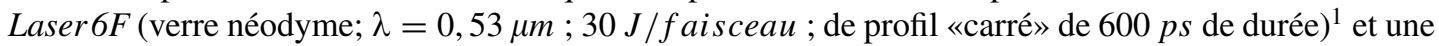
cavité cylindrique d'1 $\mathrm{mm}$ de diamètre obturée par des feuilles d'or d'épaisseur $100 \mathrm{~nm}$. L'énergie laser absorbée par ces feuilles est convertie en rayonnement $X$ qui se développe à l'intérieur de la cavité. Dans les conditions du Laser $6 F$, la température radiative de la cavité est de l'ordre de $40 \mathrm{eV}$.

Un troisième faisceau interagit avec une cible plane pour produire la source de radiographie (figure 2). La nature du matériau de cette cible annexe et l'énergie déposée est adaptée pour que le spectre de la sonde que l'on cherche à obtenir soit le plus intense et le plus «plat» possible dans la zone d'intérêt. Le signal de radiographie est analysé par un spectromètre dont le pouvoir résolvant est fourni par un cristal plan en thallium-hydrogène-phtalate (TlAP) et le spectre proprement dit est enregistré sur film. L'absorption mesurée est le rapport entre le spectre transmis et le spectre émis. Ces deux mesures peuvent être faites sur des tirs distincts obtenus dans des conditions les plus voisines possibles.

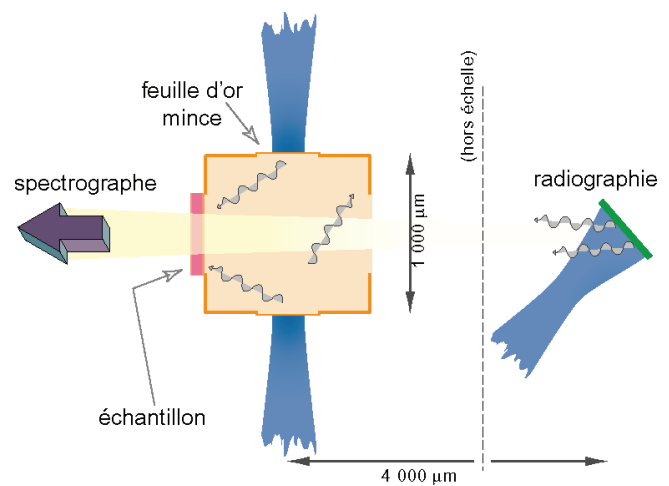

Figure 2. Le rayonnement induit par deux faisceaux laser chauffe une cavité et l'échantillon qui y est accolé. La radiographie est assurée par un troisième faisceau irradiant une cible annexe.

L'échantillon à sonder est accolé contre la cavité et chauffé par sa face tournée vers l'intérieur. Pour prévenir une détente et une chute de densité trop rapide, l'échantillon est placé entre deux feuilles qui assurent un confinement inertiel. Cet artifice doit cependant rester aussi neutre que possible vis-à-vis des aspects radiatifs, c'est à dire non seulement avoir une bonne transparence dans la gamme spectrale

${ }^{1}$ Installation démantelée en 2003. 
mesurée, mais aussi absorber globalement peu d'énergie pour ne pas être soumis lui-même à un chauffage trop important et tronquer le spectre transmis. Dans les conditions que nous rencontrons, le carbone est bien adapté car sa première discontinuité en transmission, le flanc $K$ (282 eV à température ambiante), est suffisamment à l'écart du spectre de corps noir de la cavité dont le maximum est situé vers $110 \mathrm{eV}$.

La chronologie de la radiographie doit être ajustée en fonction de deux aspects. D'une part, la proximité du maximum de l'impulsion laser correspond à des variations rapides des paramètres hydrodynamiques de la matière à sonder. D'autre part, à des instants plus tardifs les plasmas créés par les feuilles d'or impactées par le laser se rejoignent au centre de la cavité, sur l'axe de radiographie, et peuvent interférer avec la mesure. D'autres campagnes d'expériences [7] ont déterminé que cet effet survenait vers $1300 \mathrm{ps}$. En conséquence, le faisceau de radiographie est déclenché avec un retard variant entre 500 et $900 \mathrm{ps}$ (figure 3), le choix final s'appuyant sur les simulations hydrodynamiques (voir $\S$ suivant). Il a été déterminé cependant que l'émission propre de cette collision de plasma était négligeable dans les domaines spectraux considérés ici, autour de $1 \mathrm{~nm}$, ce qui autorise une mesure intégrée temporellement.

La généralisation de cette méthode de mesure des opacités à des domaines spectraux de plus basse énergie $(X U V)$ demande des précautions supplémentaires. L'émission propre de la cavité engendre un rayonnement qui devient plus ou moins superposé avec le domaine spectral d'intérêt. Il est alors nécessaire de mettre en place une mesure résolue temporellement avec l'utilisation d'une caméra à balayage de fente.

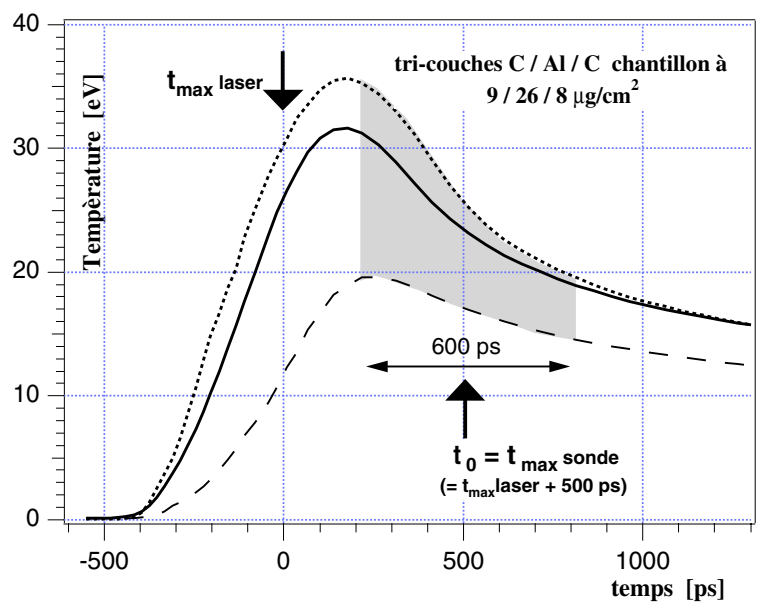

Figure 3. Évolution temporelle simulée avec MULTI de la température dans l'épaisseur d'un échantillon d'aluminium de masse surfacique totale $26 \mu \mathrm{g} / \mathrm{cm}^{2}$. Trait plein : température de la couche centrale, pointillé : la face contre la cavité, tireté : la face tournée vers l'extérieur. La période de mesure est classiquement décalée de 500 ps par rapport au maximum du laser de chauffage.

\section{SIMULATIONS HYDRODYNAMIQUES}

L'évolution spation-temporelle des paramètres du plasma dans l'échantillon est nécessaire pour analyser les résultats. En particulier, dans l'état actuel la densité électronique ne peut résulter que d'une simulation hydrodynamique concernant tout le processus de l'interaction du laser avec la cavité.

Bien que la géométrie de la cavité soit tridimensionnelle, il est concevable d'obtenir une approximation honorable en enchaînant deux simulations monodimentionnelles $1 D$. Nous utilisons pour cela le code lagrangien ID MULTI qui couple les équation de l'hydrodynamique avec celles du transfert du rayonnement [8], en utilisant un schéma numérique implicite où le rayonnement est traité selon un modèle multigroupe en fréquences et en angles. 


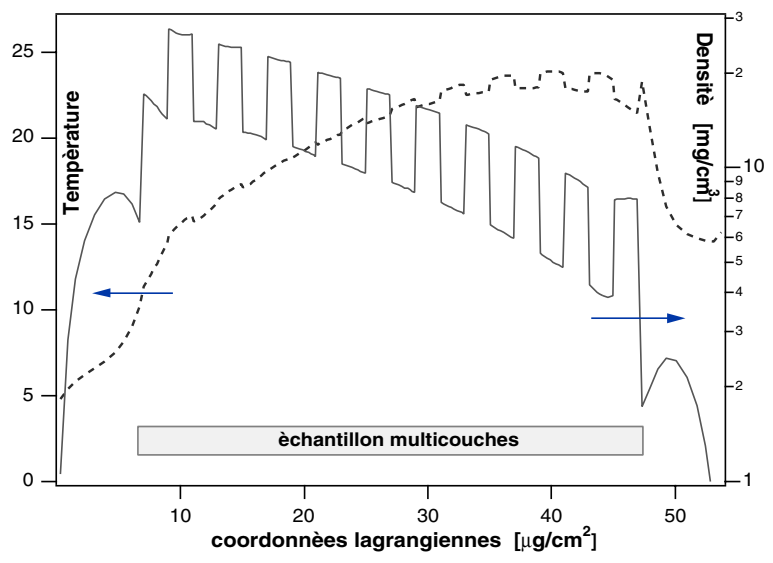

Figure 4. Profils spatiaux de température (pointillé) et de densité (trait plein) au sein d'une cible composée de 10 couches de nickel alternées avec 10 couches d'aluminium, de masse surfacique $2 \mu \mathrm{g} / \mathrm{cm}^{2}$ chacune.

Pour prendre en compte la géométrie de l'expérience avec des simulations $1 D$, nous utilisons la stratégie suivante. Tout d'abord une simulation de l'interaction du laser sur une feuille d'or de $100 \mathrm{~nm}$, avec un flux incident de $5.10^{13} \mathrm{~W} / \mathrm{cm}^{2}$, va nous fournir l'émission $\mathrm{X}$ face arrière de cette feuille, c'est à dire le flux X entrant dans la cavité. Ensuite ce flux X est lui-même la composante entrante d'une seconde simulation qui traite cette fois de la matière de l'échantillon dont on veut mesurer la photoabsorption. Le calcul fournit la densité et la température en fonction de l'axe de radiographie et du temps. Ainsi, dans notre géométrie les axes de résolution spatiale des deux simulations $1 D$ sont perpendiculaires. Passant d'un calcul à l'autre, il faut prendre en compte les effets de projection géométrique ainsi que la réémission par la paroi interne de la cavité [9].

La figure 3 présente le résultat d'une telle simulation dans l'aluminium pris entre deux couches tampons de carbone et qui montre que le profil varie sensiblement en fonction de l'espace et du temps pendant la période de mesure. La température moyenne, légèrement supérieure à $20 \mathrm{eV}$, est donc approximativement moitié de celle qui règne à l'intérieur de la cavité. Dans le cas d'une cible multicouches, soit composée de fines couches alternées d'aluminium et de nickel, nous parvenons à restreindre l'amplitude de la variation de température comme on peut le voir sur la simulation présentée sur la figure 4.

\section{PHYSIQUE ATOMIQUE}

Une des motivations du travail expérimental sur les coefficients d'absorption est de valider les codes numériques de physique atomique. Pour les plasmas constitués d'ions multi-chargés de numéro atomique assez élevé (>20, environ), la description de l'ionisation et des spectres X d'émission ou d'absorption est un problème relativement compliqué du fait du très grand nombre de niveaux atomiques en jeu. Nos mesures ont permis de tester deux approches théoriques complémentaires. La première, utilisable préférentiellement pour les élément légers comme l'aluminium, pour lesquels le nombre de transitions est de l'ordre de quelques centaines, utilise le code de physique atomique détaillée HULLAC, calcul ab-initio, basé sur un potentiel paramétrique relativiste [10]. Ce code effectue un calcul de transmission pour chaque ion considéré isolément, les abondances relatives étant supposées à l'équilibre thermodynamique (loi de Saha-Bolzmann), ou bien entrées manuellement. La deuxième approche a été développée pour décrire les éléments lourds, dont les spectres sont composés de milliers de transitions qui se superposent. Dans ce cas, on utilise une méthode de regroupement statistique des niveaux en configurations et superconfigurations (par exemple, tous les niveaux ayant un nombre fixé d'électrons dans les couches $3 s, 3 p$ ou $3 d$ ) [11]. Nous avons utilisé les résultats du code $S C O$, qui utilise cette méthode [12], et calcule la distribution ionique en 
partant d'une thermodynamique en équilibre, prenant en compte les effets d'écrantage particulièrement importants à forte densité [13]. Les transitions sont alors groupées dans des «faisceaux de transitions» gaussiens, dont on peut calculer la largeur et la longueur d'onde centrale [14]. Les calculs $S C O$ peuvent également être utilisés pour l'aluminium, et sont particulièrement bien adaptés dans le cas de fortes densité et faible ionisation [15]. Pour ces deux approches différentes, le calcul de base est effectué pour une densité et une température données. Ils peuvent aussi être couplés à un calcul hydrodynamique, en post-processeur, pour prendre en compte l'état des différentes «tranches» de matière, au prix d'un accroissement important du temps calcul [6].

\section{RÉSULTATS EXPÉRIMENTAUX}

Parmi différentes expériences récentes, nous choisissons l'exemple de la photoabsorption dans les cibles multicouches en raison de deux particularités intéressantes. D'une part comme nous l'avons vu, les gradients de densité et de température restent raisonables, et de ce fait la distribution ionique qui conditionne fortement le calcul des sections efficaces se maintient de manière assez semblable dans tout le milieu. D'autre part, la minceur des couches permet de considérer que dans deux couches successives la température est très voisine et de ce fait la spectroscopie de l'aluminium nous permet, par l'ajustement du spectre expérimental avec une simulation SCO, de confirmer la température des deux milieux. En effet, aux densités considérées, la transition $1 s-2 p$ dans l'aluminium est très sensible à l'état de charge du plasma et donc à la température. Par contre, le spectre varie lentement avec la densité que l'on fixe à la valeur donnée par la simulation hydrodynamique. Sur la figure 5-a, on représente un spectre expérimental de photoabsorbtion de l'aluminium, sur lequel on a superposé un calcul $S C O$ ajusté au mieux par une variation paramétrique de la température électronique, ici $21 \mathrm{eV}$.
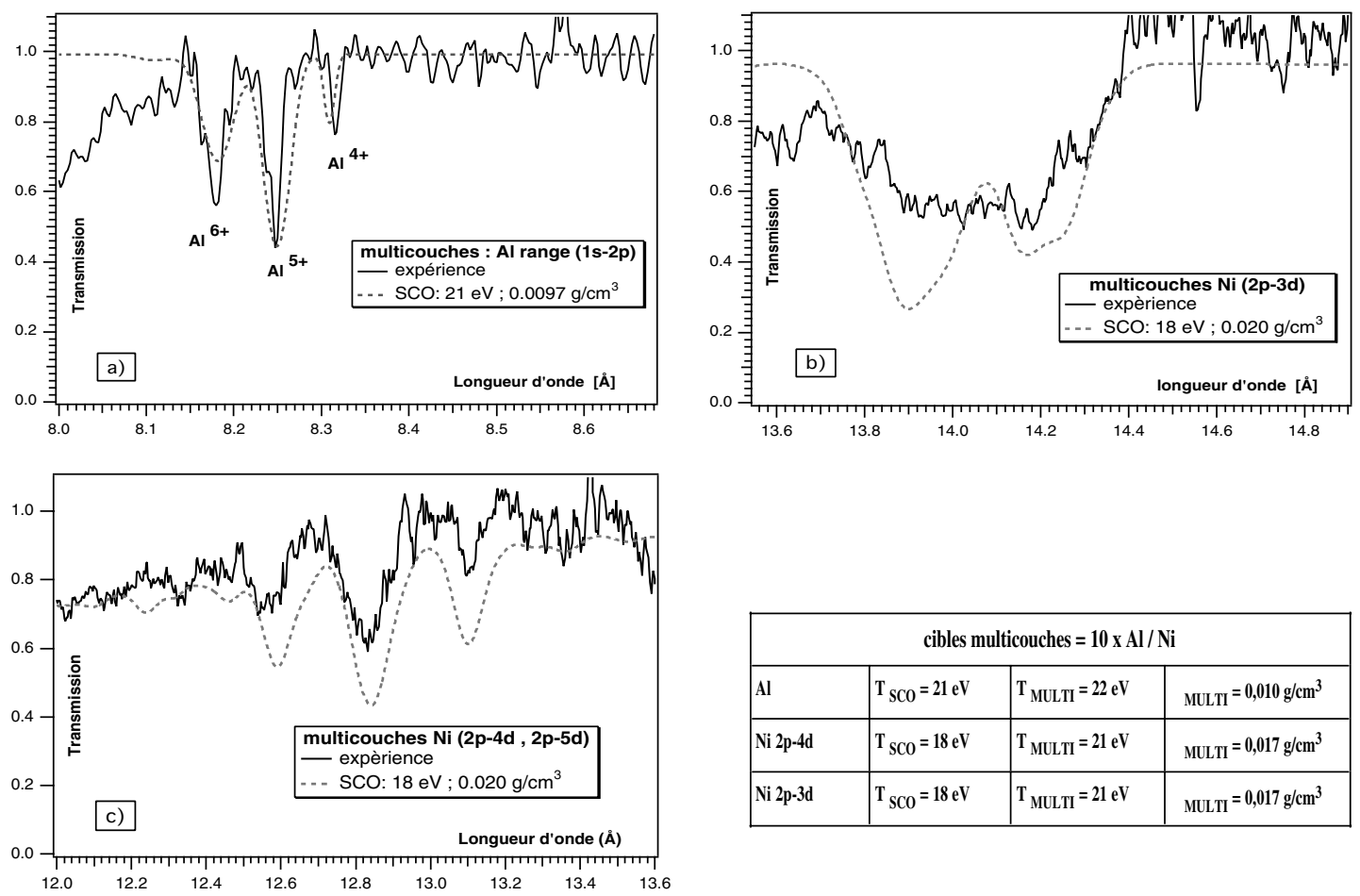

\begin{tabular}{|l|l|l|l|}
\hline \multicolumn{4}{|c|}{ cibles multicouches $=10 \times \mathrm{Al} / \mathrm{Ni}$} \\
\hline Al & $\mathrm{T}_{\mathrm{SC} 0}=21 \mathrm{eV}$ & $\mathrm{T}_{\text {MULTI }}=22 \mathrm{eV}$ & MULTI $=0,010 \mathrm{~g} / \mathrm{cm}^{3}$ \\
\hline Ni 2p-4d & $\mathrm{T}_{\text {SC0 }}=18 \mathrm{eV}$ & $\mathrm{T}_{\text {MULTI }}=21 \mathrm{eV}$ & MULTI $=0,017 \mathrm{~g} / \mathrm{cm}^{3}$ \\
\hline Ni 2p-3d & $\mathrm{T}_{\text {SCO }}=18 \mathrm{eV}$ & $\mathrm{T}_{\text {MULTI }}=21 \mathrm{eV}$ & MULTI $=0,017 \mathrm{~g} / \mathrm{cm}^{3}$ \\
\hline
\end{tabular}

Figure 5. Courbes de transmissions expérimentales et théoriques dans le domaine de la transition $1 s-2 p$ de l'aluminium (a), $2 p-3 d$ du nickel (b), $2 p$ - $4 d$ et $2 p-5 d$ du nickel (c), et comparaison des températures déterminées d'une part au moyen de la photoabsorption et d'autre part avec la simulation hydrodynamique (tableau). 
Ce «thermomètre» apparaît assez précis, puisque même une variation de $1 \mathrm{eV}$ (non représentée ici) a des effets significatifs sur l'équilibre du spectre. Sur le tableau de la figure 5, on reporte les températures comparées de la simulation hydrodynamique et du calcul de physique atomique des trois courbes des figures 5. On voit donc un bon recoupement des valeurs pour les transitions issues de l'aluminium, et légèrement décalé pour les données concernant le nickel, corps à numéro atomique plus élevé. Il faut signaler que ce désaccord est plus accentué dans le cas de cibles monocouches où les gradients du plasma sont plus marqués [16]. Sur le spectre $2 p$ - $4 d$ du nickel (figure 5-c), on remarque plus particulièrement que l'intégrale théorique de l'absorption est minorée par rapport à l'expérience. Ce problème a déjà été observé dans d'autres cas et un phénomène de saturation est proposé pour l'interpréter [17].

\section{CONCLUSION ET PERSPECTIVES}

Dans le contexte, nous considérons que l'accord théorie-expérience est satisfaisant. Cependant les gradients du plasma peuvent affecter significativement les calculs de physique atomique, à cause de la sensibilité naturelle de la distribution ionique vis-à-vis de la température et des phénomènes apparaissant à «haute» densité. De ce fait, les schémas expérimentaux où il est possible de produire des plasmas de plus grands volumes et plus homogènes sont un atout majeur pour progresser dans la précision des observations. Nous comptons par ailleurs utiliser des faisceaux sonde à durée d'impulsion significativement plus courte que les faisceaux laser de chauffage, permettant d'avoir un «instantané» favorable à la déconvolution des mesures. Sur un autre plan, l'exploration de domaines de températures et de densités plus vastes, doit mettre à l'épreuve les modèles de physique utilisés. Les grands lasers en cours d'installation en France ( $L U L I 2000, L I L$, et $L M J)$, ou à l'étranger, sont un atout pour parvenir à cet objectif .

\section{Bibliographie}

[1] C. Chenais-Popovics, Laser \& Particle Beams, 20, p. 291 (2002).

[2] S. Turck-Chièze \& al., T. Sol. Phys. 175, 247 (1997).

S. Turck-Chièze \& al., Surprising Sun, Phys.Rev.Lett., accepted 2004.

[3] M. Sako \& al., Astronom. Astrophys. 365L, 168-173 (2001).

[4] C. Chenais-Popovics \& al., Astrophys. J. Suppl. Ser. 127, 275-281 (2000).

[5] D.R. Kania \& al., Phys. Rev. A, 46,12 (1992).

[6] F. Thais \& al., J. Quant. Spectrosc. Radiat. Transfer 81, 473 (2003).

[7] C. Chenais-Popovics \& al., JQSRT 71 (2001).

[8] R. Ramis, R. Schmalz, J. Meyer-ter-Vehn, Com. Phys. Comm. 49 (1988), 475-505.

[9] F. Gilleron, Ètude du transfert radiatif et de l'opacité d'un plasma créé par rayonnement X, thèse École Polytechnique, 2000.

[10] Luc-Koenig E., Physica Scripta 62, 393 (1972)

M. Klapish, J.L. Schwob, B.S. Fraenkel \& J. Oreg, J. Opt. Soc. Am., 67, 148 (1977)

A. Bar-Shalom \& al, J. Quant.Spectr. Radiat. Transfer 71 (2001).

[11] Bar-Shalom A. \& al., Phys. Rev. A, 40, 3183 (1989).

[12] T. Blenski, A. Grimaldi \& F. Perrot, Phys. Rev. E, 55, R4889-4892 (1997).

[13] T. Blenski, A. Grimaldi, \& F. Perrot, JQSRT 65, 90 (2000).

[14] J. Bauche \& C. Bauche-Arnoult, Laser Interactions with Atoms, Solids, and Plasmas (ed. R.M. More, Plenum Press, NewYork, 1994), p. 325.

C. Bauche-Arnoult, J. Bauche \& M. Klapisch, Phys. Rev. A 31, 2248 (1985).

[15] J.-C. Pain, T. Blenski, J. Quant. Spectr. Radiat. Transfer, 81, XXX (2002).

[16] F. Thais \& al., IFSA-2001 conference, Kyoto 9-14 septembre 2001. Elsevier 2002, p. 451-455.

[17] C. Chenais-Popovics \& al., Phys. Rev. E 65, 016413-1 (2001). 